\title{
Una singolare bottega di lapicidi bizantini attiva a Hierapolis Efeso e Sardi*
}

a Claudia

\begin{abstract}
Upon the background of the study of Byzantine sculpture of the Justinian age ( $6^{\text {th }}$ century), the present survey focuses on a group of sculptures, partly unpublished, which came to light in recent archaeological campaigns carried out on the basilica complex dedicated to Apostle Philip in Hierapolis. Such materials reveal stylistic features similar to those of some fragments coming from the neighboring areas and from the city of Sardis. An exceptional element of comparison is represented here by the large crosses carved on the columns of the Basilica of San Giovanni in Ephesus. This homogeneous group of marble artifacts and their iconographic and executive characteristics are an indicator of the stylistic originality of a workshop mainly active in Hierapolis, but also testify to the mobility of masons and materials in some of the main Christian places of worship of the $6^{\text {th }}$ century.
\end{abstract}

Nel presente studio si vuole porre l'attenzione su una bottega di lapicidi finora sfuggita a una vera e propria classificazione e identificazione, e attestata in almeno tre principali città cristiane nell'area centro-occidentale dell'Asia Minore, Hierapolis di Frigia, Sardi ed Efeso (FIG. 1). Si tratta di un'indagine maturata in seno allo studio di un gruppo di reperti marmorei rinvenuti presso la Basilica di San Filippo a Hierapolis - per lo più frammenti di lastre di recinzione, pilastrini, parapetti d'ambone - accomunati da un singolare motivo decorativo che costituisce, a mio avviso, la cifra stilistica di una specifica officina attiva principalmente, se non esclusivamente, in due dei maggiori santuari dell'Asia Minore: quello dedicato alla memoria dell'apostolo Filippo, martirizzato a Hierapolis (Pamukkale) ${ }^{1}$ nel I secolo d.C., e quello

\footnotetext{
* Il presente saggio rappresenta una versione rivista e ampliata del contributo presentato al convegno organizzato dall'Università di Oslo presso l'Istituto Norvegese di Roma il 28 e 29 novembre 2016 e intitolato "Patron, Context and Style" curato dalla Prof.ssa Siri Sande che ringrazio vivamente per i proficui scambi avuti in quella circostanza. Questa stesura tiene conto inoltre dei risultati discussi successivamente in occasione della Second Annual Conference on Byzantine and Medieval Studies, svoltosi a Nicosia (Cipro) dal 12 al 14 gennaio del 2018.

${ }^{1}$ Sulla città di Hierapolis e sui monumenti bizantini si veda D'Andria 2003; Arthur 2006; 1'Atlante archeologico pubblicato da D'Andria, Scardozzi, Spanò nel 2008. Sull'eccezionale rinvenimento della basilica dedicata all'apostolo Filippo si segnala il fondamentale saggio di D'Andria 2011-2012, con le prime considerazioni sulla scoperta archeologica avve-
} 
altrettanto celebre intitolato all'evangelista Giovanni (il Teologo) ad Efeso (Seçuk). ${ }^{2}$ Partendo dallo studio dei materiali ierapolitani ${ }^{3}$ è stato possibile, in un secondo tempo, ampliare la ricerca anche ai manufatti rinvenuti durante le numerose ricognizioni condotte nella regione e finalizzate al reperimento di materiale di confronto. La basilica di San Filippo ${ }^{4}$ venne edificata sui resti della tomba del santo ubicata a sua volta nella necropoli nord-ovest della città. ${ }^{5}$ L'edificio si trova ad una quota leggermente più bassa rispetto al vicino complesso del Martyrion, ${ }^{6}$ al quale è collegato attraverso una scala monumentale. Grazie al rinvenimento di un cospicuo numero di frammenti marmorei dal cantiere del San Filippo si è potuto tentare un più sistematico studio della scultura e delle botteghe di lapicidi attivi nella città di Hierapolis, e riconsiderare, di conseguenza, la sfera di influenza e la mobilità delle maestranze locali nelle aree geografiche limitrofe.

La ricorsiva presenza di un singolare elemento "iconografico", costituito da un particolare tipo di croce latina a braccia espanse con estremità perlinate dalla caratteristica forma triangolare è all'origine di queste considerazioni.

La croce di cui ci occupiamo è collocata, in alcuni casi, su un globo e su gradini viene realizzata incisa o scolpita in un rilievo piatto emergente di pochi millimetri dal fondo. Il motivo cruciforme è ovviamente uno dei più comuni nel repertorio di forme cristiane di epoca tardo antica e bizantina, e direi il più frequente nell'insieme di forme decorative degli edifici bizantini, in particolare, poi, in età tardo-antica e giustinianea. ${ }^{7}$

Tuttavia, tra le differenti tipologie della croce presenti sui marmi del San Filippo (FIG. 2), quella senz'altro più originale nel suo assetto - e certamente la meno conosciuta - sembra essere proprio la croce latina con le estremità fortemente patenti e "perle" stilizzate in forma triangolare poste agli angoli di ciascuna terminazione (FIG. 3). Un dettaglio formale geometricamente definito che ben si associa al rilievo piatto e alle forme fortemente lineari dei de-

nuta nel 2012, e quelli di Caggia 2014 e 2016. Per un inquadramento relativo allo sviluppo del cristianesimo nella città di Hierapolis si vedano anche i contributi di Huttner 2013; sulle evidenze epigrafiche: Ritti 2017 e Filippini 2018.

${ }^{2}$ Sul San Giovanni a Efeso: Soteriou 1924; Keil, Hörmann, Soteriou 1951; Foss 1979. Per l'architettura cfr. Plommer 1962, De Bernardi Ferrero 1983 e ancora Castelfranchi Falla 1999. Da ultimo, si vedano i contributi di Büyükkolanc1, Matern 2000, Thiel 2005 e Russo 2010.

${ }^{3}$ Il ricco materiale scultoreo del San Filippo è oggetto di studio da parte di chi scrive e della collega Manuela De Giorgi. I risultati preliminari su singoli aspetti della comune ricerca sono stati presentati in occasione di diversi convegni nazionali e internazionali: De Giorgi 2016; Pedone 2016; De Giorgi 2018; De Giorgi, Pedone 2018). Sono invece in corso di pubblicazione il catalogo delle sculture bizantine (De Giorgi, Pedone c.d.s.) e Pedone c.d.s.

${ }^{4}$ D’Andria 2011-2012; Caggia 2016.

${ }^{5}$ Sulla necropoli cfr. D’Andria 2017.

${ }^{6}$ Verzone 1960; D’Andria 2011 (con bibliografia precedente); Gümgüm 2012.

${ }^{7}$ Podskalsky 1991 e Karpozilos, Cutler 1991; Heid 2006. Cfr. anche gli atti del convegno dedicato a questo soggetto: La croce. Iconografia e Interpretazione 2007. Sul motivo della croce scolpita e incisa sulle colonne si veda pure il saggio di taglio divulgativo di Moutsopoulos 2004. Sarebbe ovviamente del tutto pleonastico e inutile dare qui conto delle innumerevoli occorrenze di tale motivo iconografico, e possiamo limitarci a ricordare che le varianti, quando opportunatamente valutate nel contesto archeologico di riferimento, in rapporto alle eventuali epigrafi compresenti e alle peculiarità stilistiche, possono contribuire a suggerire o orientare la cronologia del pezzo. D'altra parte, in alcuni casi la croce rappresenta il principale, se non quasi esclusivo elemento decorativo, e basta a questo riguardo - tralasciando i casi di epoca iconoclasta (cfr. Moorhead 1985) - citare l'esempio più celebre della Santa Sofia giustinianea, dove la croce viene reiterata sia sui mosaici (Mango 1962) sia nell'arredo scultoreo (cfr. Guiglia Guidobaldi, Barsanti 2004). Un studio interessante sulle varianti iconografiche della croce, sebbene riferito a tutt'altro ambito geografico, è quello di Ballardini 2004, 165-192. 
cori a cui è frequentemente associato nelle lastre del San Filippo. Non mi sembra dunque implausibile ipotizzare che questa così originale configurazione possa essere un vero e proprio "marchio di fabbrica" di una specifica bottega attiva appunto in questa regione dell'Asia Minore nel corso del VI secolo. ${ }^{8}$

La deliberata deviazione da un modello o da una "regola stilistica" dominante - regola talmente diffusa da non dover essere qui nemmeno discussa - si trasforma quindi in un dato visivo emergente, soprattutto se direttamente confrontato con il modello stesso, vale a dire la croce a estremità patenti tipica dell' età giustinianea ${ }^{9}$ (FIG. 4). Questo dato o, potremmo meglio dire, questa "esuberanza" creativa vincolata, che poi si esplica anche attraverso il ductus tecnico-esecutivo, coinvolge pure gli aspetti sottesi al discorso sulla produzione ed esportazione dei manufatti lapidei, e tocca dunque il ben noto problema dei rapporti tra centro e periferia dell'impero, tra modelli e creazioni locali, soprattutto in un arco cronologico che, come quello del VI secolo, è stato spesso considerato troppo marcatamente "unitario". A tale proposito, basti citare il significativo esempio di alcuni frammenti emersi nello scavo del San Filippo, tutti riconducibili a un'unica lastra di marmo bianco decorata con il motivo delle losanghe concentriche, la quale - proprio in ragione delle caratteristiche cromatiche e tattili, nonché del motivo decorativo - poteva essere facilmente scambiata per una lastra di marmo proconnesio di provenienza costantinopolitana, ma che si è rivelata essere, grazie alle analisi petrografiche, un pezzo di marmo locale estratto a pochi chilometri a nord-est della città ${ }^{10}$ (Mermer Tepe).

Tra i frammenti di lastre ricomposte che presentano la decorazione in questione, la più interessante è una grande lastra, ${ }^{11}$ molto probabilmente usata come parapetto delle gallerie, caratterizzata da un semplice schema tripartito profilato da listelli piatti (FIG. 5). Nei riquadri laterali della lastra, delimitati da un doppio listello circolare, trova posto il motivo del fiore a dodici petali dal profilo allungato, ma scolpiti alternando pieni e vuoti così da creare l'effetto ottico - grazie al cosiddetto "completamento amodale" - di un disegno a doppio registro da cui emerge la figura di un esagono. Nel rettangolo centrale (FIG. 6), invece, due croci simme-

\footnotetext{
${ }^{8}$ Le fasi della basilica del San Filippo sono chiaramente sintetizzate in D'Andria 2011-2012 e Caggia 2016. A una prima monumentalizzazione della tomba del santo, avvenuta nel corso del III-IV secolo, è seguita una prima costruzione basilicale nel V secolo e un successivo rifacimento nel corso del VI, con una cesura importante costituita dal terremoto del 674 i cui danni si registrano anche in molti altri edifici della città. Nel corso del periodo post-giustinianeo si registrano diversi interventi di modifica della struttura, con il rifacimento pavimentale e la parziale ri-decorazione dell'edificio. Sebbene siano attestati interventi di manutenzione cittadina durante l'età di Giustiniano, la totale assenza di fonti dirette che indichino un preciso intervento di rinnovo della basilica del San Filippo da parte di questo imperatore, lascia aperta la possibilità che i lavori di ridecorazione siano avvenuti in momento successivo alla sua morte (565) per opera di uno dei suoi successori. Cfr. supra.

${ }^{9}$ Sappiamo quanto l'esportazione dei marmi fosse ampiamente praticata nel periodo giustinianeo (Barsanti 1989), e come essa fosse strettamente collegata al potere imperiale centrale. Per Giustiniano, l'edificazione di nuovi complessi e il relativo invio di materiale scultoreo dalla capitale costituiva il "segno" di un preciso investimento ideologico oltre che puramente materiale. L'ampiezza dell'argomento e delle indagini su singole aree geografiche preclude qui una sintesi esaustiva, pertanto si rimanda alle pubblicazioni di Barsanti 1989; Sodini 1989; Sodini, Barsanti, Guiglia Guidobaldi 1998. Ancora più recentemente sul tema dell'esportazione vedi Russo 2007; Pensabene, Barsanti 2008; Barsanti, Paribeni 2016 anche per gli ampi riferimenti bibliografici.

${ }^{10}$ Vedi Caggia 2016, 477, fig. 6 (H13_221), 478, tab. 4; De Giorgi 2016, 496, fig. 16; e in particolare, per una più ampia descrizione del pezzo, della tipologia decorativa e dei suoi modelli, Pedone in De Giorgi, Pedone 2018, 380-382, tav. 5, fig. 7a-c.

${ }^{11}$ La lastra H13_218/219 è di marmo Docimio (Caggia 2016, 478, tab. 4, fig. 8; De Giorgi 2018, 297-298, fig. 3 b.
} 
triche sono poste ai lati di un disco definito anch'esso da due listelli piatti e decorato al suo interno con un motivo a quincunx. Le croci sono a loro volta collegate al disco centrale attraverso i due lemnisci, che terminano con due grandi foglie cuoriformi. Il rilievo è omogeneo ed emerge da fondo di pochi millimetri (circa $0.7-1 \mathrm{~cm}$ ). L'osservazione ravvicinata e le immagini a luce radente permettono di cogliere meglio i segni di lavorazioni a gradina, le linee incise e i punti usati per traferire il disegno sul marmo. Le croci sono contraddistinte da terminazioni accentuate ed espanse, con i piccoli triangoli posti su tutte le terminazioni dei bracci.

Sebbene la struttura tripartita degli elementi del riquadro centrale, come pure la presenza dei lemnisci, si ispirino a una tradizione ben nota e di ampia circolazione in età giustinianea, $\mathrm{i}$ lapicidi che hanno scolpito e concepito questa e altri frammenti simili, pur attingendo al modello predominante, lo reinterpretano in maniera significativa, sia modificando la forma della croce sia prediligendo una maggiore stilizzazione e astrazione dello spazio decorativo.

Lo stesso tipo di croce si ritrova su una ulteriore lastra frammentaria di grandi dimensioni, che doveva presentare un analogo schema decorativo tripartito, ma di cui resta solo la parte destra, integra, e una piccola porzione di quella centrale ${ }^{12}$ (FIG. 7). In questo caso, il motivo cruciforme si trova incorniciato al di sotto di un arco sostenuto da due colonne poggianti su gradini. ${ }^{13}$ Diversamente dalla lastra precedente, la croce si erge su un globo, a sua volta collocato su tre gradini. Nonostante la porzione conservata sia esigua, non c'è dubbio circa le terminazioni triangolari tipiche dello schema che stiamo qui analizzando. ${ }^{14}$

È stato possibile rintracciare un analogo disegno compositivo su una lastra rettangolare in marmo bianco conservata presso il Museo archeologico di Hierapolis (FIG. 8), la cui provenienza resta comunque ignota, seppure riconducibile all'ambito ierapolitano o ad aree limitrofe. ${ }^{15}$ La prolungata esposizione all'aperto del pezzo ne ha in parte compromesso lo stato di conservazione, rendendo difficile l'identificazione della tipologia marmorea (marmo bianco locale?). Lo stile appare piuttosto rozzo e meno ricercato che nella lastra del San Filippo, soprattutto se si osserva proprio il dettaglio delle terminazioni perlinate triangolari. La presenza delle colonnine tortili e dei due elementi vegetali laterali, nonché lo stile più approssimativo, lasciano ipotizzare una sorta di "imitazione" degli esemplari del San Filippo, forse con una cronologia posteriore alla seconda metà del VI secolo, o da collocarsi piuttosto all'inizio del VII.

Tornando ai reperti della basilica dell'apostolo Filippo, vale la pena considerare ancora cinque frammenti che ricompongono parzialmente una lastra decorata con elementi simili ai precedenti esemplari, ma secondo uno schema differente ${ }^{16}$ (FIG. 9). Entro un motivo centrale quadrilobato delimitato da un doppio listello liscio è scolpita la croce perlinata su gradini; nelle porzioni laterali, al quadrilobo si annodano tre elementi, di cui quello mediano circolare

\footnotetext{
${ }^{12}$ De Giorgi 2018, 297, fig. 3a; Pedone in De Giorgi, Pedone 2018, 386-387, tav. 9, fig. 13.

${ }^{13}$ Il numero di gradini su cui si innesta il braccio verticale non è fisso e può variare: i casi qui presentati variano da un minimo di due gradini a un massimo di cinque.

${ }^{14} \mathrm{La}$ lastra conserva in alto l'ampia cimasa su cui è incisa l'iscrizione "KYPITZIOU". Per questa si veda Ritti 2011 2012, 58, fig. 5 .

${ }^{15}$ Parman 2002, 167-168, tav. 93, fig. 116, inv. n. 755 (con datazione VII-VIII secolo); Pedone in De Giorgi, Pedone 2018, 387, tav. IX, fig. 14 .

${ }^{16}$ Da una visione d'insieme del materiale finora studiato emerge chiaramente lo sforzo operato dalla bottega di lapicidi che realizzarono questo gruppo di sculture di sperimentare sempre nuove varianti compositive a partire da pochi ed essenziali elementi geometrici e decorativi. L'unico elemento invariante resta proprio la croce.
} 
e quelli superiore e inferiore semicircolari. Questi ultimi accolgono al loro interno, alternativamente, un motivo a girandola e fiori dai petali affusolati. Anche in questo caso il rilievo è di pochi millimetri e sono visibili le linee incise per trasferire il disegno sul marmo, così come i segni del compasso per i profili circolari della decorazione. L'accuratezza esecutiva dei doppi listelli, come pure la precisione nella definizione delle forme geometrico-vegetali, rivela un ricercato livello esecutivo, pur nella stilizzazione del disegno e negli effetti bidimensionali del decoro.

Sebbene la maggior parte di queste croci scolpite a basso rilievo ricorrano per lo più su frammenti di lastre, vi è un caso, al momento unico, in cui una di esse appare come ornamento di un parapetto di ambone conservato nei depositi del Museo Archeologico di Istanbul, ma proveniente da una delle chiese bizantine di Sardi ${ }^{17}$ (FIG. 10). Per le proporzioni del disegno, non sembrano esserci dubbi sul fatto che il pezzo sia da ascrivere alla stessa bottega dei plutei del San Filippo.

Una diversa e più corsiva configurazione della nostra croce, incisa invece che in rilievo (FIG. 11), è stata rintracciata infine sul bordo sferico del pomello di uno dei grandi pilastrini probabilmente in opera nella solea della basilica del San Filippo, il cui stilobate è ancora in situ lungo la navata centrale. La croce è realizzata a fianco di una croce a rilievo più semplice e il pomello, dal profilo piuttosto schiacciato, presenta un ampio foro quadrangolare destinato a ospitare la grappa metallica che lo univa probabilmente a un pomello più piccolo posto alla sommità. ${ }^{18}$ Anche in questa curiosa variante incisa è presente la forma della croce latina con perline triangolari sulle braccia patenti. Per ritrovare un significativo confronto dobbiamo tornare tra i materiali del Museo di Hierapolis, dove è stato possibile individuare un grande sarcofago frammentario in marmo bianco, ${ }^{19}$ decorato sui lati brevi da due croci incise della medesima forma ${ }^{20}$ (FIG. 12). I triangoli terminali sono marcatamente accentuati e suggeriscono, anche in questo caso, una sorta di "marchio di fabbrica".

Tuttavia, i casi forse più noti della versione "incisa" della nostra croce si trovano lontano da Hierapolis, ossia sulle colonne di marmo proconnesio del complesso basilicale del San Giovanni a Efeso ${ }^{21}$. Queste croci $^{22}$ di grandi dimensioni - già in parte studiate per la presenza delle invocazioni che le accompagnano - si trovano su otto diversi fusti di colonna collocati in punti diversi della basilica (vedi qui lo schema grafico in FIG. 13). Sebbene non sia facile stabilire se l'attuale collocazione dei fusti coincida di fatto con la posizione originaria o se sia invece, almeno in alcuni casi, il risultato del lavoro di anastilosi sul monumento, è comunque interessante valutare il fenomeno in sé e il significato simbolico che probabilmente sottende

\footnotetext{
${ }^{17} \mathrm{Il}$ pezzo non è attualmente nella collezione permanente del museo archeologico di Istanbul a causa dei lavori di riallestimento. Si pubblica qui la foto di William E. Bestch scattata nel 1970 e conservata presso l'archivio fotografico del Dumbarton Oaks Center di Washington e consultabile on line: http://atom.doaks.org/atom/index.php/photographs-ofarchitectural-capitals-in-istanbul-by-william-earl-betsch-1970.

${ }^{18}$ La fronte del pilastrino presenta un'ordinaria modanatura con un campo rettangolare e terminazione semilunata.

${ }^{19}$ Nello spazio superiore sinistro, tra i bracci della croce, si scorgono incise due lettere, forse una iota e un sigma lunato.

${ }^{20}$ Nella porzione superiore sinistra sono incise le due lettere IC del nome di Iesus, probabilmente completate nella parte mancante con le due lettere XP di Cristos.

${ }^{21}$ Come Giovanni, anche Filippo era denominato teologo, e benché le sue vicende agiografiche siano più complesse e si intreccino a tradizioni diverse, entrambi, insieme a san Paolo, testimoniano la diretta predicazione degli apostoli di Ges ù in Asia Minore: le "colonne", potremmo dire, della penetrazione del cristianesimo in queste regioni a partire dal I secolo, come attestato dagli Atti degli Apostoli.

${ }^{22}$ Soteriou 1924; Keil, Hörmann, Soteriou 1951, 34-35, in part. 478, nr. 10a; 12a; 13a; 14; 15a, taff. LXIV.
} 
la pratica di incidere grandi croci e invocazioni sui principali sostegni dell'edificio. Significato che potrebbe essere ulteriormente connesso all'importanza dei colonnati nell'economia generale dell'innovativa pianta del San Giovanni, che, come sappiamo, replicava peraltro quella ben più celebre dell' Apostoleion di Costantinopoli.

Come ho potuto appurare de visu, le croci risultano tutte ben visibili all'interno dello spazio sacro: la loro altezza oscilla dai 40 ai $60 \mathrm{~cm}$ e sono tutte per lo più orientate verso il naos o lo spazio centrale del transetto, oppure - ancora più significativamente - poste ad incorniciare uno dei portali laterali di accesso della basilica (nel caso delle colonne nn. 1-2). Si tratta dunque di collocazioni facilmente individuabili, che evidentemente miravano a rendere "pubblico" il contenuto dell'invocazione, come pure il nome del "richiedente". ${ }^{23}$ Non è però neppure da sottovalutare l'aspetto prettamente ornamentale, che spiegherebbe la reiterazione dello stesso schema quando non addirittura delle stesse dimensioni. ${ }^{24}$ Il disegno è identico a quello delle croci ierapolitane, con l'unica differenza che quelle di Efeso sono incise e non a rilievo (FIG. 14) e in almeno due occorrenze una piccola croce greca è ulteriormente inserita all'interno del globo (FIG. 14a-b). Ma l'elemento più cospicuo è comunque la presenza delle invocazioni, ${ }^{25}$ incise su due o tre righe tra gli spazi di risulta dei bracci della croce. Le lettere greche hanno caratteri epigrafici simili e variano solo leggermente nella loro altezza, mentre le frasi ripetono la formula canonica dell'invocazione e intercessione rivolta al Signore (kyrie boethei) o allo stesso San Giovanni, seguita poi dal nome del fedele o dell'individuo a cui viene estesa la preghiera. In due occorrenze è indicata anche la professione e il particolare gesto votivo, come nel caso del "servo Giorgio", che ha fatto erigere la colonna su cui è incisa la croce, oppure del "falegname Giovanni", che ha contribuito alla costruzione dell'edificio (vedi sotto, colonne nn. 2 e 7).

Nel corso di una più recente visita al monumento ho potuto poi verificare nuovamente l'esatta ubicazione delle croci sui fusti delle colonne e raccogliere nuovi dati di confronto, anche alla luce della ricerca sui pezzi ierapolitani, che erano stati finora valutati solo parzialmente. Vale quindi la pena analizzare partitamente la casistica, tenendo conto della posizione dei singoli pezzi, come riportato nello schema della FIG. 14:

Colonna 1 (FIG. 14, 1 e FIG. 15): la colonna è ubicata davanti ad uno dei portali laterali sul lato sud, in corrispondenza della prima campata occidentale. Il fusto è quello di sinistra e va considerato insieme alla colonna n. 2, poiché entrambe inquadrano uno degli accessi alla navata laterale e al naos. La croce, molto rovinata e parzialmente abrasa nella parte centrale, si innesta su un "piede" della stessa larghezza del braccio verticale $(2 \mathrm{~cm} \mathrm{ca}$.) a sua volta collegato a un globo del diametro di $7 \mathrm{~cm}$. All'interno del disco circolare trova posto una piccola croce greca incisa con quattro bracci dalle terminazioni espanse. Il globo poggia a sua volta su cinque gradini. Non si conserva nessuna iscrizione, ma non è da escludere che ve ne fosse una, analogamente alla colonna n. 2, perduta a causa della sfaldatura o abrasione del marmo. Diversi graffiti di lettere greche appena percepibili compaiono anche sul resto del

\footnotetext{
${ }^{23}$ Le croci si trovano tutte a un'altezza di circa $50 / 70 \mathrm{~cm}$ dalla base delle colonne.

${ }^{24}$ La stretta affinità formale delle croci rivela a mio avviso una medesima cronologia. Tuttavia dal punto di vista epigrafico l'uso della iota con il doppio puntino può far pensare ad una datazione posteriore all'età giustinianea. Tuttavia, la parzialità del numero complessivo delle croci rimaste e la possibile aggiunta delle iscrizioni anche in momento successino non cambiano la questione più generale dello stile specifico e identitario delle croci e della loro funzione. Cfr. supra. ${ }^{25}$ Cfr. Keil, Hörmann, Soteriou 1951, 278-279, taf. LXIV, nr. 10a, 12a, 13a, 14, 15a.
} 
fusto a formare delle invocazioni, ${ }^{26}$ e va segnalata infine un'ulteriore croce leggermente incisa entro un doppio cerchio, finora non documentata ${ }^{27}$ (FIG. 16).

Colonna 2 (FIG. 14, 2): la colonna è posta a destra della precedente e utilizzata per inquadrare uno dei due portali (quello a occidente) che si aprono lungo il fianco sud dell'edificio. La croce è posta a circa $1,20 \mathrm{~cm}$ dalla base del fusto, ripete esattamente il disegno precedente, ma è meglio conservata. È alta, comprese le terminazioni perlinate, circa $23 \mathrm{~cm}$ e larga 14.4 $\mathrm{cm}$ (nell'apertura del braccio orizzontale); il globo ha un diametro di circa $\mathrm{cm} \mathrm{7;} \mathrm{i} \mathrm{gradini} \mathrm{alla}$ base sono quattro. Ben leggibili sono le lettere greche, alte circa 1.4/1.5 cm, che compongono l'invocazione e sono impaginate su due linee, divise tra i quattro bracci della croce. Si legge:

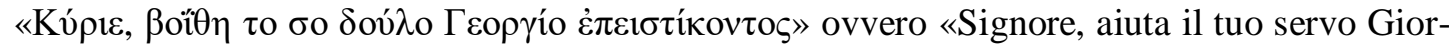
gio, poiché ha posto [questa colonna]». ${ }^{28}$

Colonna 3 (FIG. 14, 3 e FIG. 17): la croce orientata verso l'interno del naos. Le dimensioni sono maggiori rispetto alle due precedenti: l'altezza, comprese le terminazioni perlinate, è di circa $46.5 \mathrm{~cm}$, mentre il braccio orizzontale è largo $27.5 \mathrm{~cm}$. Lo spessore dei bracci è di circa $3 \mathrm{~cm}$. Il diametro del globo è di $8.5 \mathrm{~cm}$. All'incrocio dei bracci vi è un incavo circolare con quattro ulteriori piccoli fori, forse funzionali al fissaggio di una placchetta metallica. ${ }^{29}$

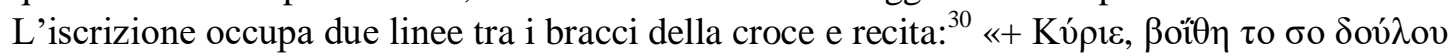
'Ioóvov $\xi v \lambda \imath v \alpha »$, ovvero «Signore, aiuta il tuo servo, falegname [del tempio] di Giovanni».

Colonna 4 (FIG. 14, 4 e FIG. 18): la croce è incisa a circa $55 \mathrm{~cm}$ dalla base del secondo fusto del colonnato nord, nella prima campata occidentale del naos. La parte terminale della croce è perduta, tuttavia le dimensioni sembrano ripetere perfettamente quelle della croce sul colonnato opposto (n. 3). L'altezza attuale della parte conservata è di circa $44 \mathrm{~cm}$ e la larghezza del braccio orizzontale $27 \mathrm{~cm}$. I bracci misurano $3 \mathrm{~cm}$ di spessore; il diametro del disco è di 8 $\mathrm{cm}$ e i gradini sono cinque. Anche in questo caso le lettere incise occupano due linee, di cui

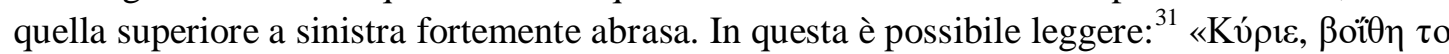

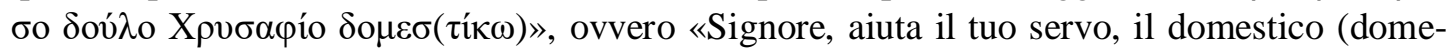
stikos) Crisafio».

Colonna 5 (FIG. 14, 5 e FIG. 19): La croce è incisa sulla prima colonna del transetto nord ed è rivolta verso l'interno della campata. L'altezza della croce è di circa $43 \mathrm{~cm}$, mentre il braccio orizzontale è largo $24 \mathrm{~cm}$ circa. Lo spessore dei bracci è di $3.5 \mathrm{~cm}$; il globo ha un diametro di $\mathrm{cm} 8$ e i gradini su cui si innalza la croce sono cinque. La superficie è leggermente

\footnotetext{
${ }^{26}$ Ivi.

${ }^{27}$ Oltre alla suddetta croce greca, segnalo anche i profili stilizzati di animali, forse cervi, incisi con un tratto incerto e puntellato sulla terza colonna della campata occidentale, nel lato sud, e le numerosissime piccole croci rintracciabili sulla maggior parte dei fusti e dei blocchi marmorei disseminati nel complesso.

${ }^{28}$ Una trascrizione in greco della medesima si trova in Keil, Hörmann, Soteriou 1951, 278, nr. 10a. Ringrazio la collega e amica Chiara Bordino per il suo prezioso aiuto nella traduzione italiana di questa e delle altre iscrizioni qui riportate.

${ }^{29}$ La pratica di inserire piccole reliquie nelle colonne è attestata anche nella Santa Sofia, come recentemente osservato da Natalia Teteriatnikov 1998.

${ }^{30}$ Cfr. Keil, Hörmann, Soteriou 1951, 278, nr. $12 a$.

${ }^{31}$ Cfr. Keil, Hörmann, Soteriou 1951, 279, nr. $13 a$.
} 
abrasa nella parte centrale, ma non compromette la lettura del profilo inciso della croce. Nella parte inferiore sono visibili le linee guida funzionali al trasferimento del disegno sul marmo e il foro per la punta del compasso utilizzata per tracciare la circonferenza del globo. Non c'è nessuna iscrizione.

Colonna 6 (FIG. 14, 6 e FIG. 20): la croce è incisa su una delle quattro colonne del transetto nord più esterne. Come nel caso precedente, non compare nessuna iscrizione. La croce è alta circa $37.5 \mathrm{~cm}$, con un'apertura del braccio orizzontale di $28.5 \mathrm{~cm}$. Il diametro del globo è di circa $6.7 \mathrm{~cm}$. La porzione superiore è in parte abrasa o leggermente scalpellata.

Colonna 7 (FIG. 14, 7 e Fig. 21): la croce si trova sul primo fusto del colonnato posto sul lato nord, che delimita lo spazio del bema. È la sola ad essere rivolta a est. La croce misura $29 \mathrm{~cm}$ circa di altezza e 17.5 di larghezza del braccio orizzontale; lo spessore dei bracci varia da $2.3 \mathrm{~cm}$ (braccio orizzontale) a 2.5 (verticale); il diametro del globo è di $7.4 \mathrm{~cm}$ e i gradini sono quattro. L'iscrizione, a circa $60 \mathrm{~cm}$ dalla base del fusto, è disposta su quattro linee di cui tre nella porzione superiore del braccio orizzontale e una nella parte inferiore. Vi si legge: ${ }^{32}$

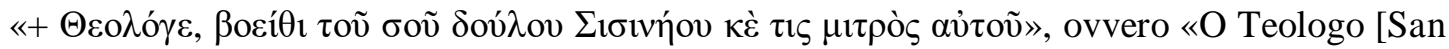
Giovanni], aiuta il tuo servo Sisinnio e sua madre».

Colonna 8 (FIG. 14, 8 e FIG. 22): la colonna su cui è incisa la croce giace a terra insieme ad altri frammenti marmorei davanti al pilatro sud-ovest del transetto sud. Purtroppo l'iscrizione non è visibile, ma è stata trascritta da Hörmann nel 1951 e come la precedente è posta su tre

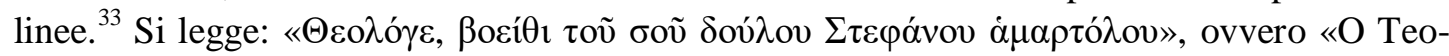
$\operatorname{logo}$ (San Giovanni), aiuta il tuo servo, il peccatore Stefano».

Le dimensioni, la posizione e la cura dell'incisione nell' esecuzione di queste croci suggerisce l'importanza simbolica ad esse riservata e costituisce una prova indiretta dell'importanza della bottega chiamata a realizzarle. Non si tratta, infatti, di dettagli marginali, come possono essere i graffiti. ${ }^{34}$ Se nei contenuti, negli intenti devozionali e sotto il profilo documentale i due tipi di tracce, incise e graffite, possono essere considerati sullo stesso piano, dal punto di vista della loro "visibilità", degli effetti sulla decorazione, nonché della prassi esecutiva, ci sono notevoli differenze. La realizzazione di tali incisioni, infatti, deve aver certamente coinvolto non solo il ruolo dei benefattori della chiesa - come il domestikos Crisafio o il già citato "servo Giorgio", che probabilmente finanziò l'acquisto di una o più colonne - ma anche quello delle autorità religiose, che autorizzarono dei lapicidi professionisti, o una vera e propria bottega, a inserire queste invocazioni in luoghi eminenti e simbolicamente importanti, come sono appunto i sostegni stessi della basilica. Di là dalle loro funzioni materiali ed architettoniche, le colonne della chiesa avevano fin dalle origini, come è ben noto, una valenza simbolica e metaforica: ${ }^{35}$ richiamo ai santi pilastri del tempio di Salomone (Re, VII, 13-23),

\footnotetext{
${ }^{32}$ Cfr. Keil, Hörmann, Soteriou 1951, 279, nr. 14.

${ }^{33}$ Cfr. Keil, Hörmann, Soteriou 1951, 279, nr. 15a.

${ }^{34}$ Numerosi sono i graffiti - non tutti facilmente leggibili - rintracciabili sui fusti delle colonne di marmo proconnesio della basilica e dell'antistante quadriportico e compaiono anche sulle colonne decorate con le grandi croci incise. Cfr. Josef Keil in Keil, Hörmann, Soteriou 1951, 278-279 e ss. che però non tiene conto dei graffiti figurati.

${ }^{35}$ Vedi tra gli altri il classico saggio di Krautheimer 1993, 111 e ss.
} 
figura dei dodici apostoli e sostegno vivente del corpo della chiesa terrena. Dunque luoghi e oggetti fortemente connotati da un punto di vista liturgico e devozionale. È perciò da escludere che le incisioni di cui ci stiamo occupando siano semplicemente i gesti estemporanei o "furtivi" di devoti e pellegrini occasionali desiderosi di lasciare un qualche segno votivo del proprio passaggio. Piuttosto, queste croci sembrano rappresentare una sorta di privilegio esclusivo concesso soltanto a coloro che ne erano "degni" per qualche ragione, e si potrebbe persino supporre che le colonne prive di iscrizione fossero ancora "in attesa" di accogliere il tributo di un donatore.

Tenendo conto di questi aspetti, è plausibile pensare che un simile compito possa essere stato affidato a una specifica bottega (o a un particolare lapicida), forse efesina o di origine ieropolitana e affermatasi anche a Efeso, che conia un proprio "modello" poi reiterato nella regione e non attestato altrove. Un modello che diventa quindi quasi espressione singolare di una peculiarità geografica, e forse non inconsapevole di un'esplicita autonomia, che pur rielaborando stimoli esterni "inventa" tuttavia una forma relativamente nuova - un marchio unico.

È auspicabile che il procedere delle indagini archeologiche in questi siti e nei territori limitrofi possa portare alla luce ulteriori testimonianze che possano a loro volta arricchire il piccolo corpus di pezzi marmorei qui considerati per la prima volta tutti insieme, e a mio avviso importanti per comprendere più realisticamente la molteplicità delle direzioni su cui si sviluppò la scultura bizantina delle provincie.

Silvia Pedone

Sapienza Università di Roma

silvia.pedone@gmail.com 


\section{BIBLIOGRAFIA}

Arthur, P. 2006: Hierapolis (Pamukkale) Bizantina e Turca. Guida archeologica, Istanbul.

Ballardini, A. 2004: "Le croci del «Convento Rosso»: una nota preliminare", in Progetto pilota Deir el Ahmar Deir anba Bishoi "Convento Rosso", introduzione di S. Casartelli, Roma, 165-192.

Barsanti, C. 1989: 'L'esportazione di marmi dal Proconneso nelle regioni pontiche durante il IV-VI secolo", RIASA III, 12, 91-220.

Barsanti, C., Paribeni, A. 2016: "La diffusione del marmo Proconnesio nelle Marche in età classica e paleocristiana: il ruolo del porto di Ancona", Hortus Artium Medievalium 22, 200-214.

Büyükkolanc1, M., Matern, P. 2000: Das Leben des Heiligen Johannes und die Johanneskirche, Selçuk.

Caggia, M.P. 2014: "La collina di S. Filippo a Hierapolis di Frigia: osservazioni sulle fasi di occupazione bizantina e selgiuchide (IX-XIV sec.)", ScAnt 20.2, 143-161.

Caggia, M.P. 2016: "Prime indagini sul terrazzo dell'Aghiasma: la chiesa di San Filippo", in F. D’Andria, M.P. Caggia, T. Ismaelli (a cura di), Hierapolis di Frigia VIII, 1-2. Le attività delle campagne di scavo e restauro 2007-2011, , Istanbul, II, 729-765.

Castelfranchi Falla, M. 1999: "Il complesso di San Giovanni ad Efeso nel quadro dell'architettura giustinianea dell'Asia Minore", in R. Pillinger, O. Kresten, F. Kritznger, E. Russo (hrsg.), Efeso paleocristiana e bizantina /Frühchristliches und byzantinisches Ephesos, Vienna, 89-98.

D’Andria, F. 2003: Hierapolis di Frigia (Pamukkale). Guida Archeologica, Istanbul.

D'Andria, F. 2011: "Conversion, Crucifixion and Celebration. St Philip's Martyrium at Hierapolis", Biblical Archaeology Review 37, 34-46.

D’Andria, F. 2011-2012, "Il Santuario e la tomba dell'Apostolo Filippo a Hierapolis di Frigia", RendPontAc 84, 1-52.

D'Andria, F. 2017: "The Sanctuary of St Philip in Hierapolis and the tombs of saints in Anatolian cities", in J.R. Brandt, E. Hagelberg, G. Bjørnstad, S. Ahrens (ed.), Life and Death in Asia Minor in Hellenistic, Roman, and Byzantine Times. Studies in Archaeology and Bioarchaeology, Oxford, 3-18.

D’Andria, F., Scardozzi, G., Spanò, A. 2008: Atlante di Hierapolis di Frigia (Hierapolis di Frigia II), Istanbul.
De Bernardi Ferrero, D. 1983: "San Giovanni di Efeso", Corsi di Cultura sull'Arte Ravennate e Bizantina 30, 93-113.

De Giorgi, M. 2016: "Materials, forms and models in the St Philip church of Hierapolis: Byzantine architectural elements", in T. Ismaelli, G. Scardozzi (ed.), Ancient quarries and building sites in Asia Minor. Research on Hierapolis in Phrygia and other cities in south-western Anatolia: archaeology, archaeometry, conservation, Bari, 489-500.

De Giorgi, M. 2018: "Divine liturgy and human skills in the architectural sculpture from the church of the Apostle in Hierapolis (Phrygia)", in C. Şimşek, T. Kaçar (ed.), Geç antik çağda Lykos vadisi ve çevresi / The Lykos Valley and Neighbourhood in Late Antiquity, Istanbul, 291-308.

De Giorgi, M., Pedone, S. 2018: "La scultura della chiesa di San Filippo a Hierapolis. Nuovi dati e il progetto Marmora Phrygiae", in S. Cosentino, M.E. Pomero, G. Vespignani (a cura di), Dialoghi con Bisanzio Spazi di discussione, percorsi di ricerca, Atti del Convegno Nazionale di studi della Associazione Italiana di Studi Bizantini (Ravenna, 22-26 settembre 2015), I-II, Spoleto, Fondazione Centro italiano di studi sull'alto Medioevo (Collana Quaderni di Bizantinistica, 19), 367-387.

De Giorgi, M., Pedone, S. c.d.s: La scultura bizantina del San Filippo di Hierapolis: il catalogo.

Filippini, A. 2018: "Concilliors, Heretics, and Archbishops in Late Antique Hierapolis: Recent Epigraphical Findings Concerning the City, Its Territory, and the History of Hierapolis' Bishopric $\left(4^{\text {th }}-9^{\text {th }}\right.$ Cent.)", in C. Șimşek, T. Kaçar (eds.), Geç antik çağda Lykos vadisi ve çevresi / The Lykos Valley and Neighbourhood in Late Antiquity, Istanbul, 250-284.

Foss, C. 1979: Ephesus after Antiquity: A late antique, Byzantine and Turkish City, Cambridge.

Guiglia Guidobaldi, A., Barsanti, C. 2004: Santa Sofia di Costantinopoli: l'arredo marmoreo della grande chiesa giustinianea, Città del Vaticano.

Gümgüm, G. 2012: Il Martyrion di Hierapolis di Frigia (Turchia). Analisi archeologica e architettonica, Oxford.

Heid, S. 2006: s.v. "Kreuz", Reallexikon für Antike und Christentum, Stuttgart, c. 21, s. 1100-1147.

Huttner, U. 2013: Early Christianity in the Lycus Valley, Leiden (Ancient Judaism and early Christianity, 85). 
Karpozilos, A., Cutler, A. 1991: s.v. "Cross in Everyday Life", The Oxford Dictionary of Byzantium, I, New York, Oxford, 551-552.

Keil, J., Hörmann, A., Soteriou, G.A. 1951: Forschungen in Ephesos, Band IV, Heft. 3, Die Johanneskirche, Wien.

Krautheimer, R. 1993: "Introduzione a un'iconografia dell'architettura sacra medievale", in Id., Architettura sacra paleocristiana e medievale e altri saggi su Rinascimento e Barocco, Torino, 98-150.

La croce. Iconografia e Interpretazione 2007: La croce. Iconografia e Interpretazione (secoli I-inizio $X V I$ ), Atti del convegno internazionale di studi (Napoli, 6-11 dicembre 1999), a cura di B. Ulianich, Napoli.

Mango, C. 1962: The Mosaics of St. Sophia at Istanbul, Washington D.C.

Moorhead, J. 1985: "Iconoclasm, the Cross and the Imperial Image”, Byzantion 55, 165-179.

Moutsopoulos, N.K. 2004: Staurōmenoi kiones / Crossed Columns, Athens.

Niewöhner, P. 2017: The Archaeology of Byzantine Anatolia. From the End of Late Antiquity until the Coming of the Turks, Oxford.

Parman, E. 2002: Ortaçă̆da Bizans döneminde Frigya (Phrygia) ve Bölge Müzelerindeki Bizans taş eserleri, Eskişehir.

Pedone, S. 2016: "Byzantine sculpture in Hierapolis: Engraving Techniques and Colour Finishes", in T. Ismaelli, G. Scardozzi (ed.), Ancient quarries and building sites in Asia Minor. Research on Hierapolis in Phrygia and other cities in south-western Anatolia: archaeology, archaeometry, conservation, Bari, 501-509.

Pedone, S. c.d.s: "New Evidence for Byzantine Sculptures from the Basilica of St Philip at Hierapolis", in D. Moreau (éds.), with the collaboration of I. Baldini, N. Beaudry, O. Heinrich-Tamáska, L. Milanović, M. Milinković, I. Popović and C.S. Snively, Archaeology of a World of Changes. Selected Papers on Late Roman and Early Byzantine Archaeology from the $23^{\text {rd }}$ International Congress of Byzantine Studies (Belgrade, 22-27 August 2016). In honorem Claudiae Barsanti, Oxford, BAR Publishing.

Pensabene, P., Barsanti, C. 2008: Reimpiego e importazioni di marmi nell'Adriatico paleocristiano e bizantino, in La cristianizzazione dell'Adriatico (Antichità Altoadriatiche 66), Trieste, 455-490.
Plommer, H. 1962: "St. John's Church, Ephesus”, AS 12, 119-129.

Podskalsky, G. 1991: s.v. "Cross", The Oxford Dictionary of Byzantium, I, New York, Oxford, 549-550.

Ritti, T. 2011-2012: “Alcune iscrizioni rinvenute nella Chiesa di S. Filippo", RendPontAc 84, Appendice I, 53-62.

Ritti, T. 2017: Hierapolis di Frigia IX. Storia e istituzioni di Hierapolis (Hierapolis di Frigia IX), Istanbul.

Russo, E. 2007: "Le maestranze greco-costantinopolitane a Roma nel VI secolo", in A.C. Quintavalle (a cura di), Medioevo Mediterraneo: l'Occidente, Bisanzio e l'Islam dal Tardoantico al secolo XII, Atti del VII Convegno Internazionale di Studi di Parma, 21-25 settembre 2004, Milano-Parma, 139-152.

Russo, E. 2010: Sulla cronologia del S. Giovanni $e$ altri monumenti paleocristiani di Efeso, Wien.

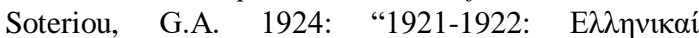

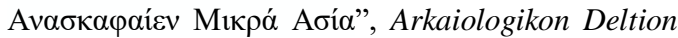
7, 89-206.

Sodini, J.-P. 1989: "Le commerce des marbres à l'époque protobyzantine", in Hommes et richesses dans l'empire byzantine (IVe-VIIe s.), I, Paris, 163 186.

Sodini, J.-P. 2000: "Le commerce des marbres dans la Méditerranée (IVe-VIIe s.)", in V Reuniò d'Arqueologia Cristiana Hispànica (Cartagena, 16-19 d'abriò de 1998), Barcelona, 423-446.

Sodini, J.-P., Barsanti, C., Guiglia Guidobaldi, A. 1998: "La sculpture architecturale en marbre au VIe siècle à Constantinople et dans les régions sous influence constantinopolitaine", in Acta XIII CIAC (Split - Poreč 1994), Città del Vaticano, 301-376.

Taş, T., Özacan, F. 2015: "Yüzıllar arasında haç motiflerinin gelișmi / The Development of Cross Motives Between Late $4^{\text {rd }}-7^{\text {th }}$ Centuries AD.", Journal of Süleyman Demirel University Institute of Social Sciences 1, 21, 247-275.

Teteriatnikov, N. 1998: "Devotional crosses in the columns and was go Hagia Sophia”, Byzantion 68, 2, 419-445.

Thiel, A. 2005: Die Johanneskirke in Ephesos, Wiesbaden.

Verzone, P. 1960: "Il Martyrium ottagono a Hierapolis di Frigia. Relazione preliminare", Palladio. Rivista di storia dell'architettura e restauro I-II, 1-20. 


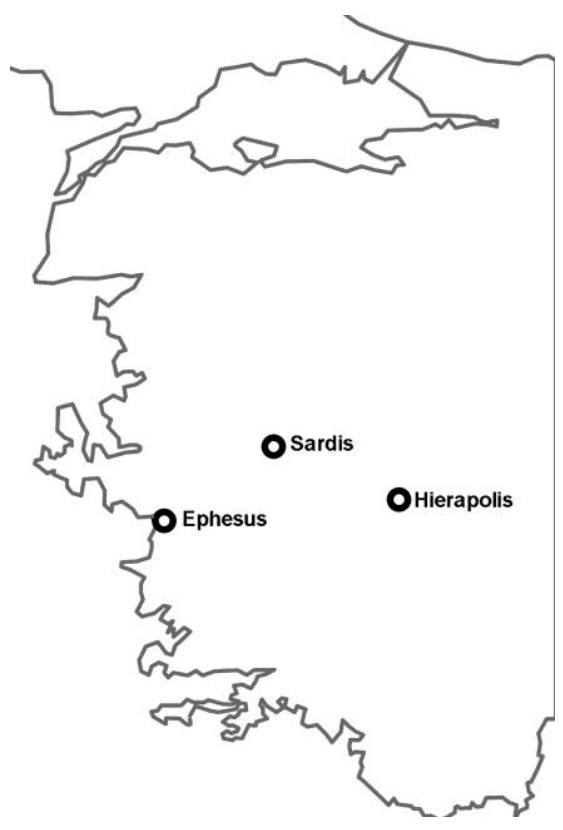

FIG. 1 - Mappa geografica con indicazione delle città di Hierapolis, Sardis e Efeso.
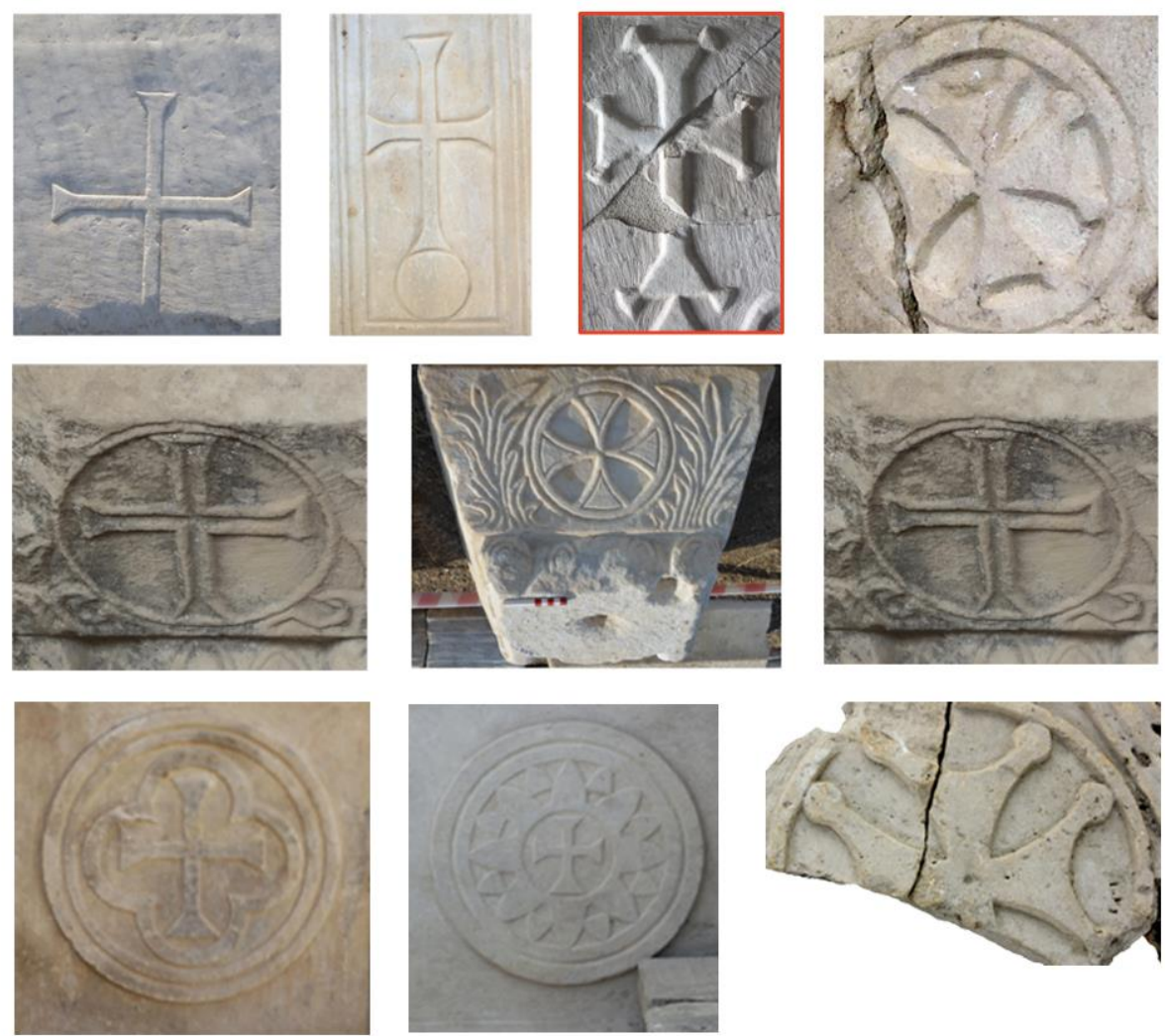

FIG. 2 - Diverse tipologie di croci rinvenute sul materiale scultoreo del San Filippo a Hierapolis (foto S. Pedone). 

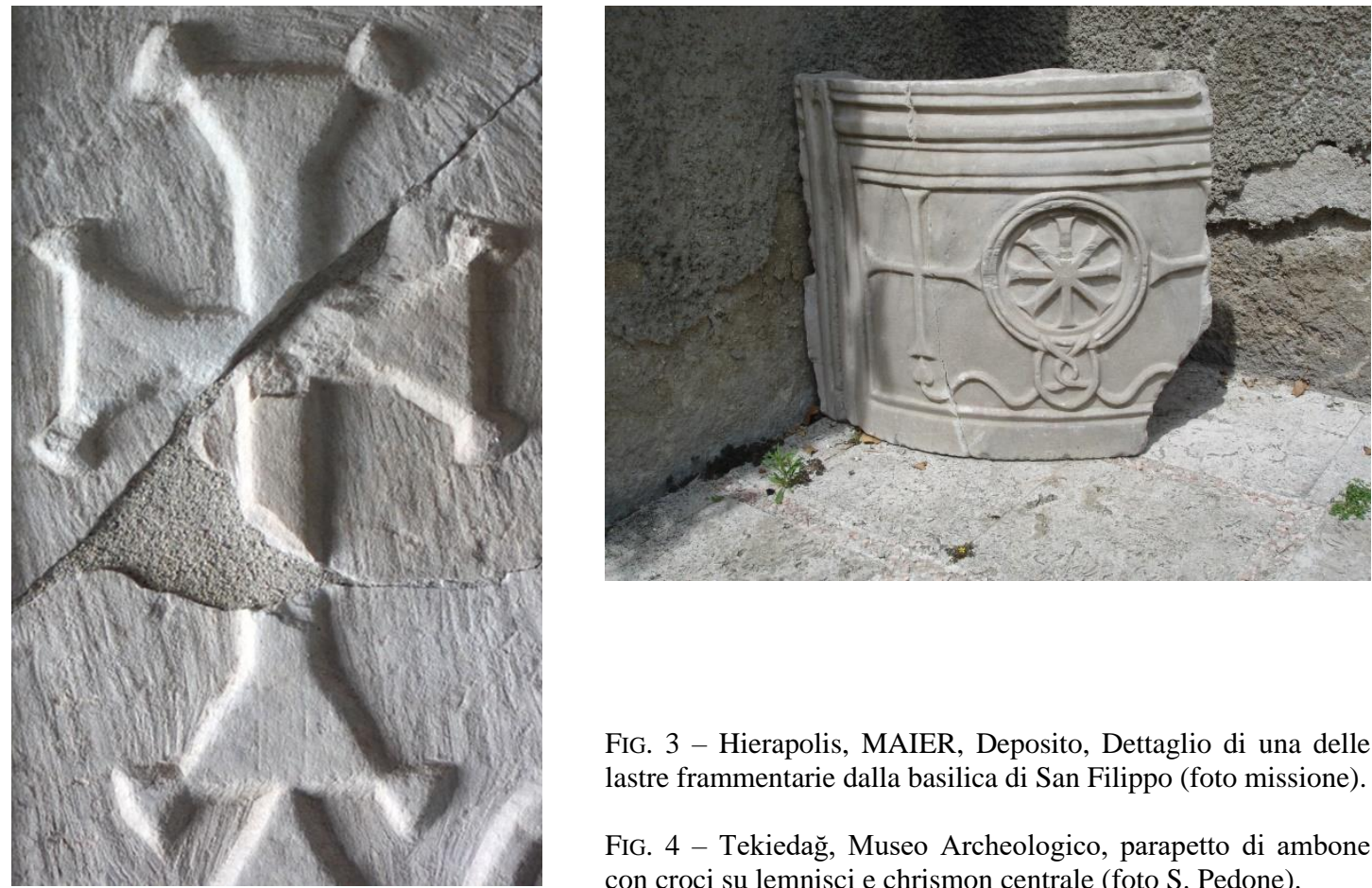

FIG. 3 - Hierapolis, MAIER, Deposito, Dettaglio di una delle lastre frammentarie dalla basilica di San Filippo (foto missione).

FIG. 4 - Tekiedağ, Museo Archeologico, parapetto di ambone con croci su lemnisci e chrismon centrale (foto S. Pedone).
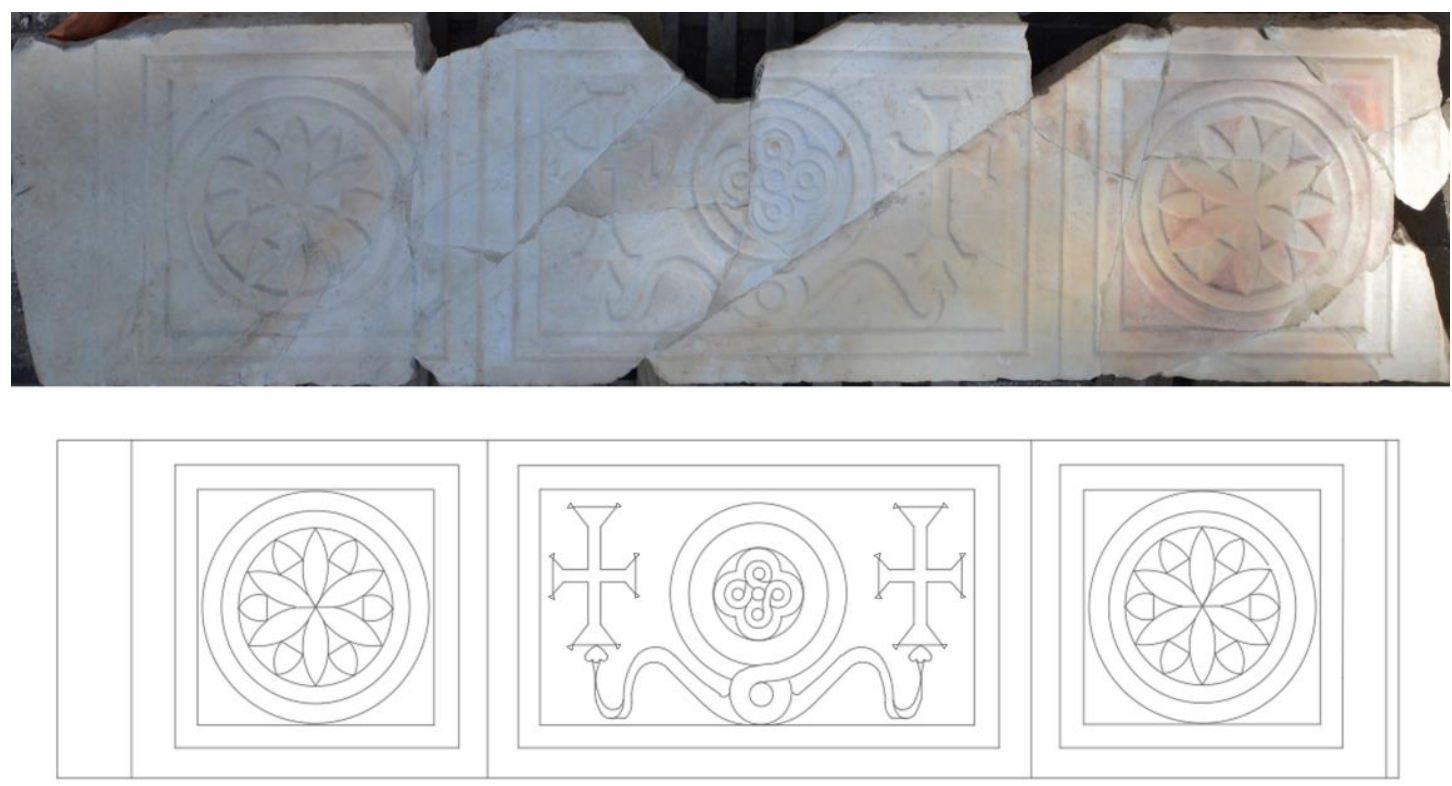

FIG. 5 - Hierapolis, MAIER, Deposito, Lastra frammentaria dalla basilica di San Filippo e ricostruzione grafica (foto De Giorgi). 


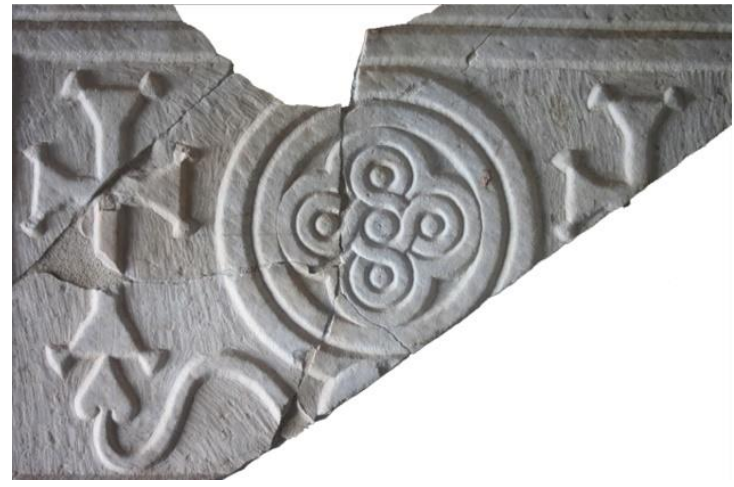

FIG. 6 - Hierapolis, MAIER, Deposito, Particolare della porzione centrale della lastra frammentaria precedente (foto missione).

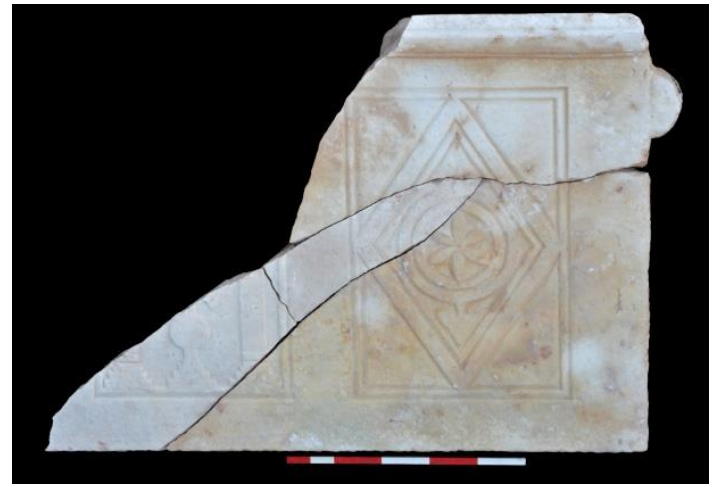

FIG. 7 - Hierapolis, MAIER, Deposito, Frammento di parapetto con cimasa (foto missione).
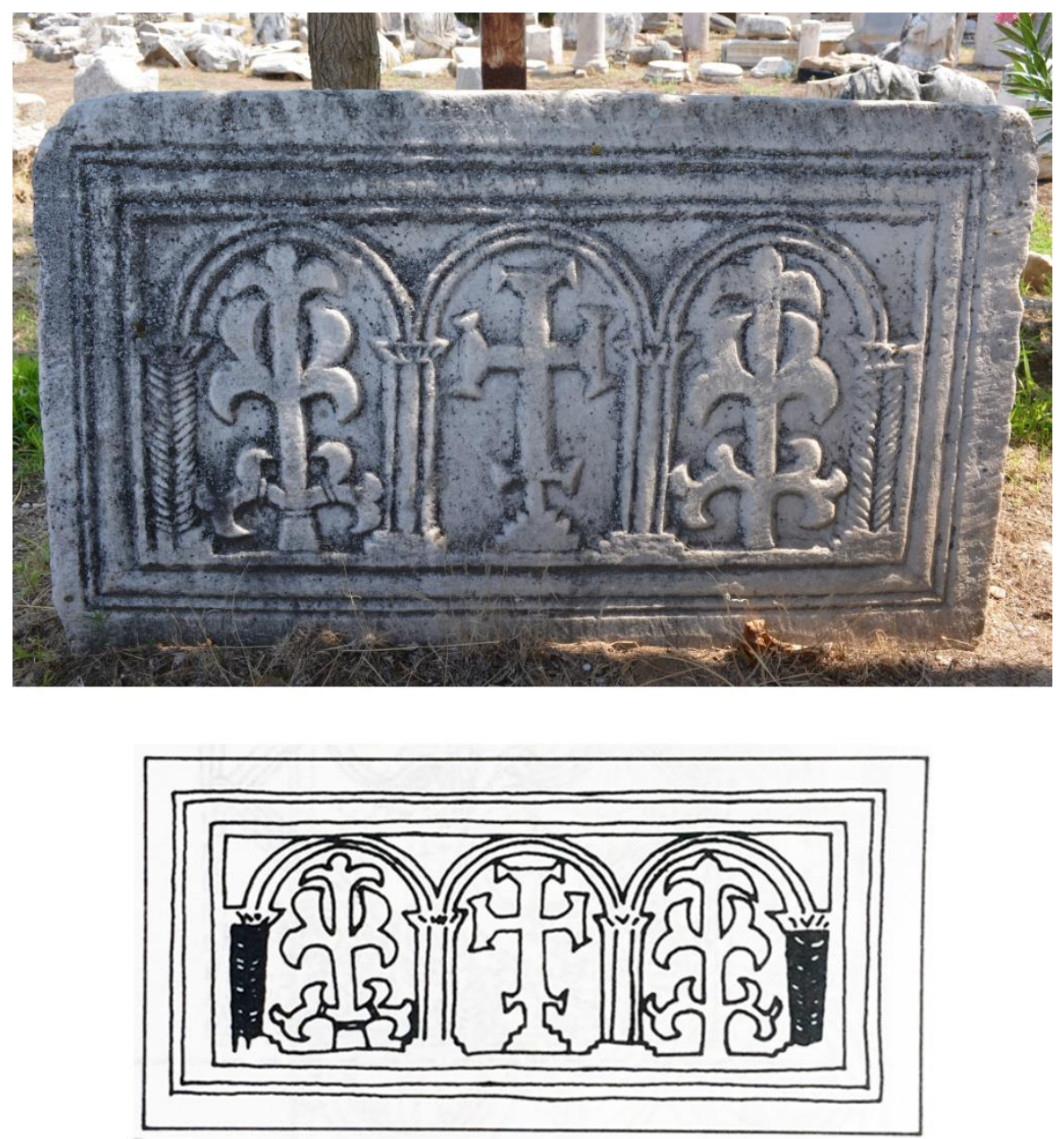

FIG. 8 - Hierapolis, Museo, Lastra di marmo bianco e ricostruzione grafica da Parman 2002 (foto S. Pedone). 

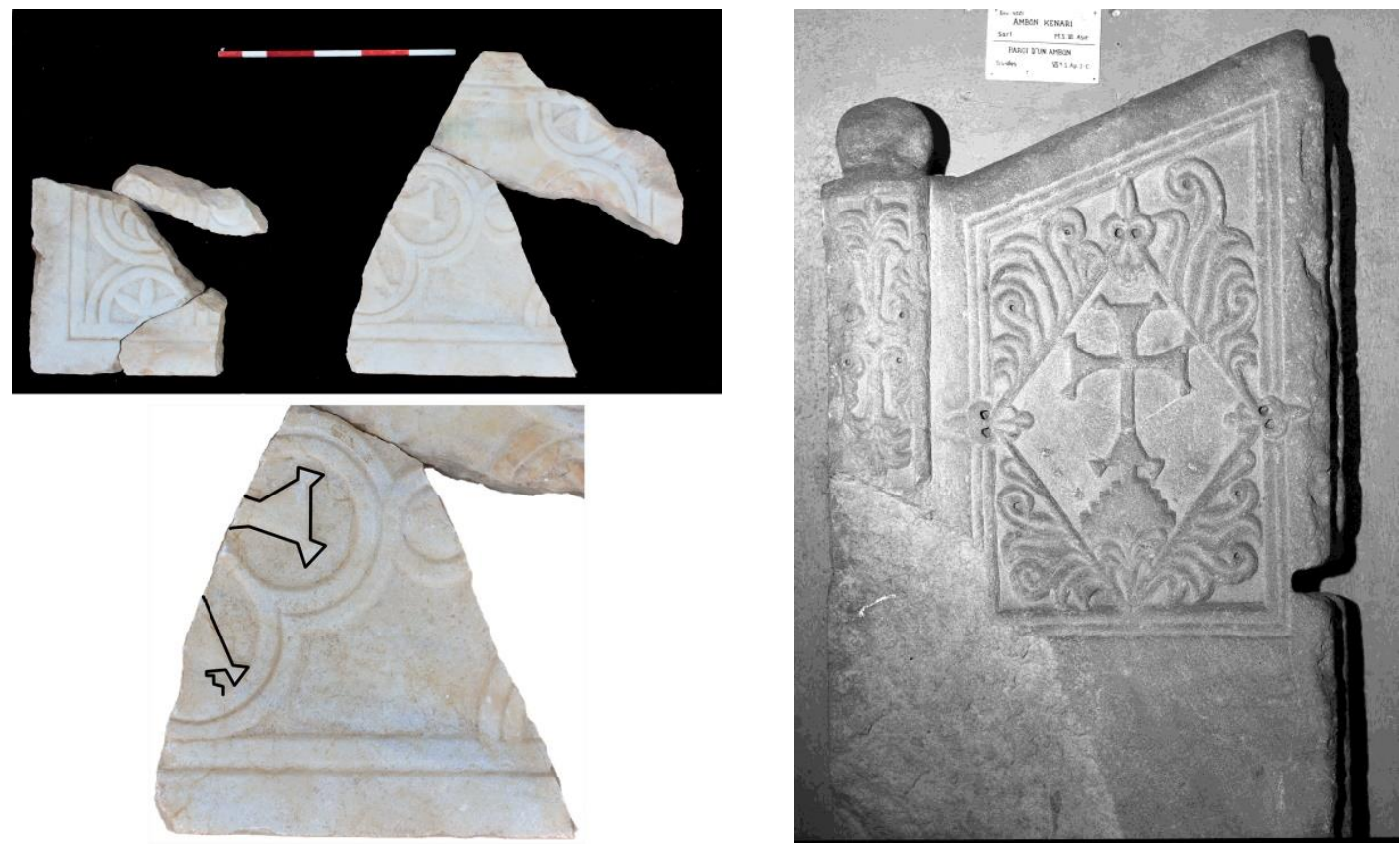

FIG. 9 - Hierapolis, MAIER, Deposito, Frammenti pertinenti ad una lastra dal San Filippo, dettaglio (foto missione).

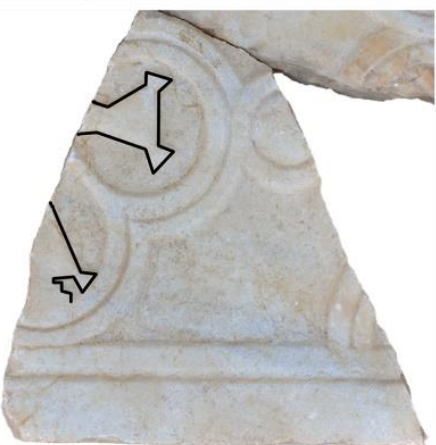

FIG. 10 - Istanbul, Museo Archeologico, Parapetto di ambone proveniente da Sardi, scatto fotografico di William Earl Betsch 1970 (PH.BZ.002, Image Collections and Fieldwork Archives, Dumbarton Oaks, Trustees for Harvard University, Washington, D.C.)
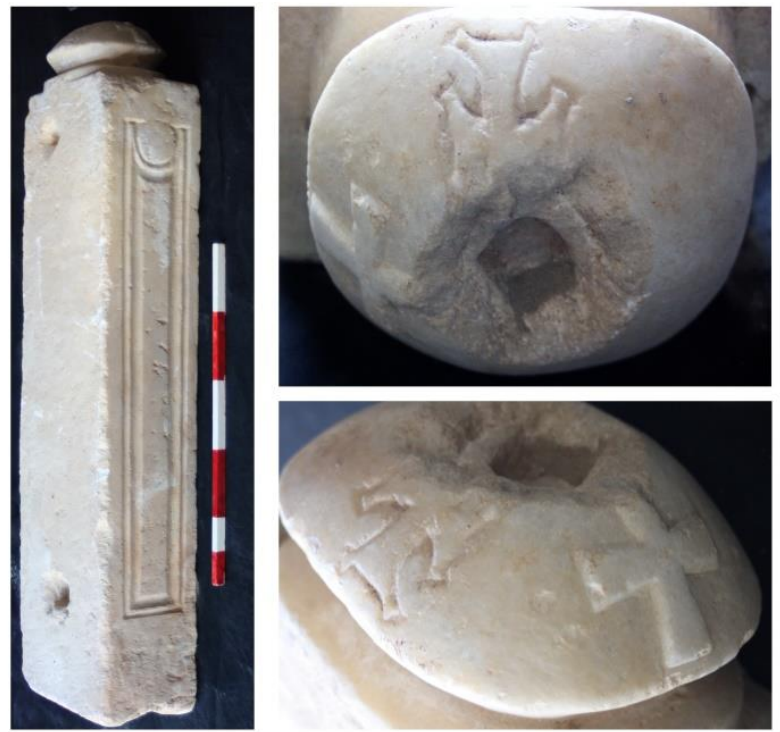

FIG. 11 - Hierapolis, MAIER, Deposito, pilastrino dal San Filippo (foto missione). 

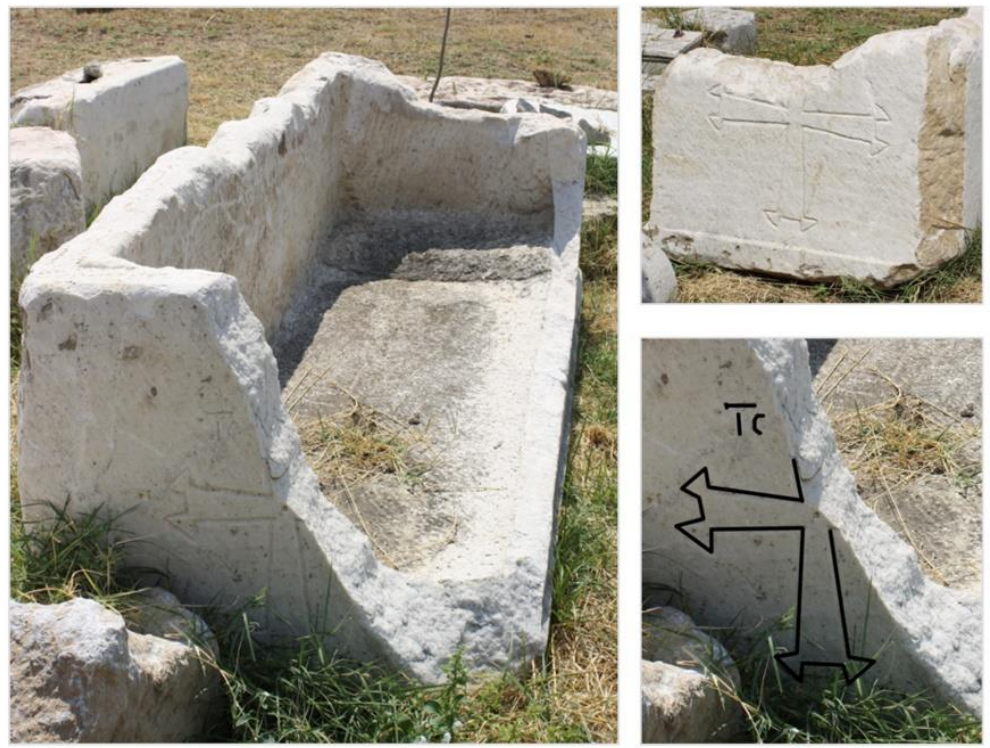

FIG. 12 - Hierapolis, Museo, Sarcofago (foto S. Pedone).

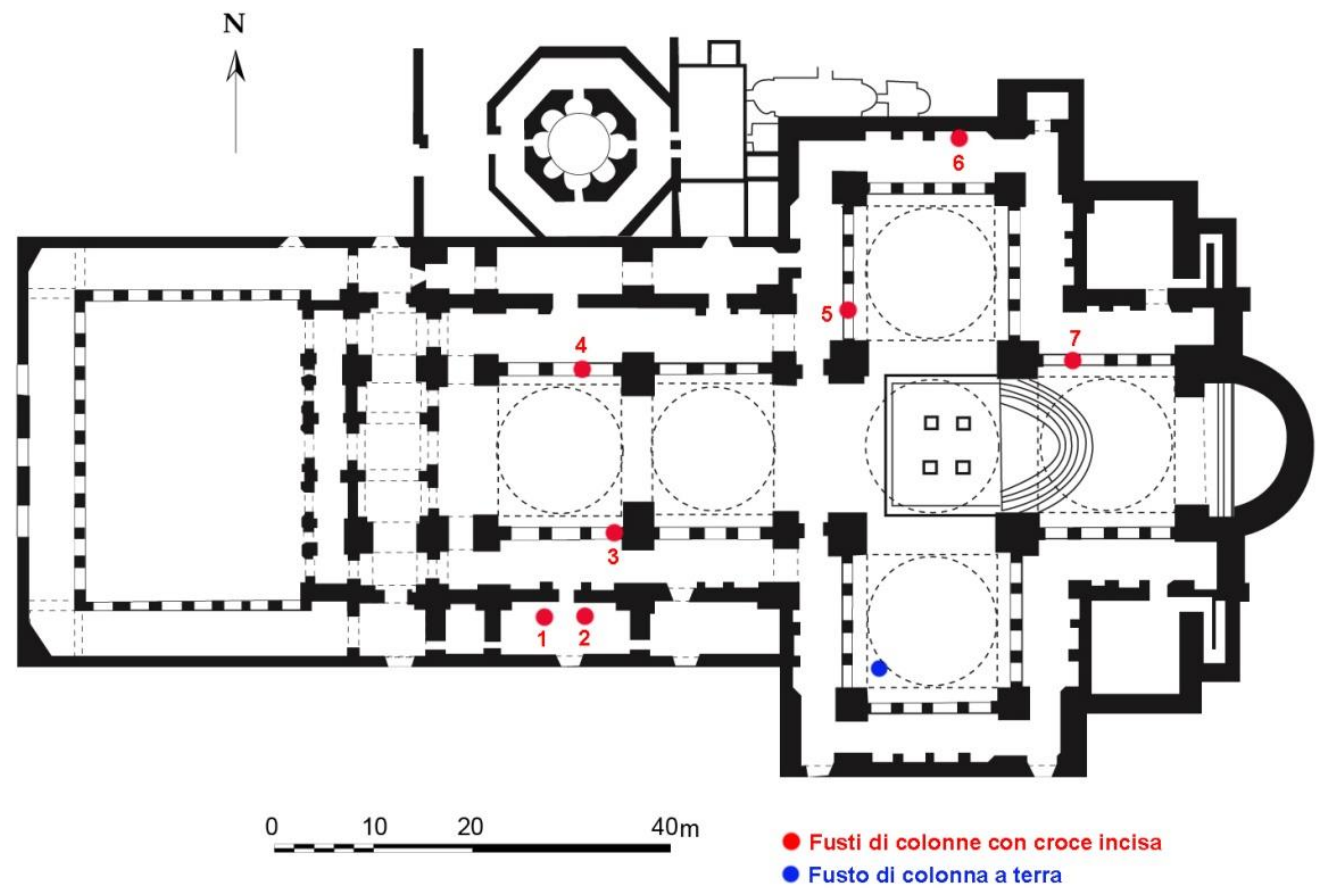

FIG. 13 - Pianta del San Giovanni di Efeso con l'indicazione delle colonne su cui sono incise le croci (grafica S. Pedone). 


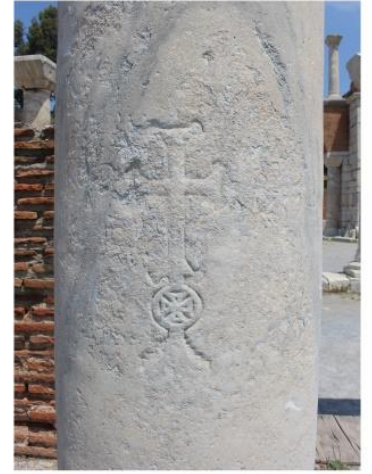

1

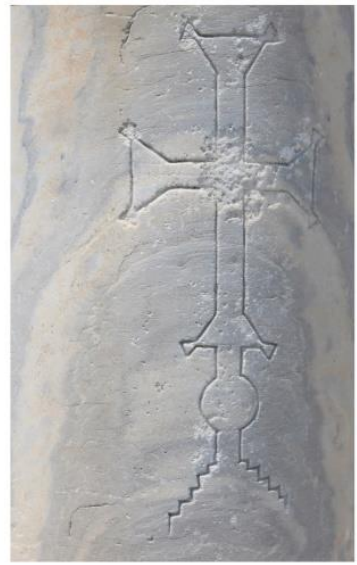

5

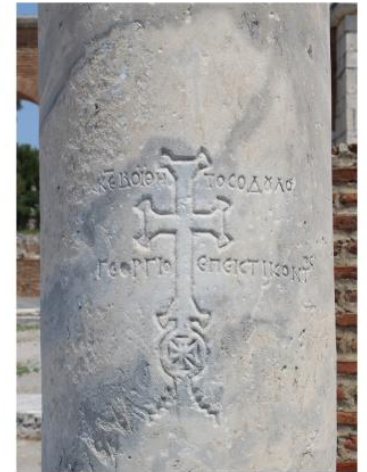

2

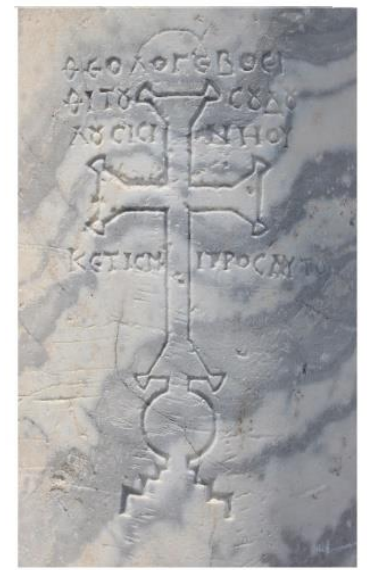

6

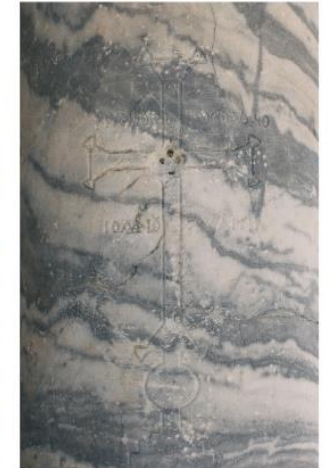

3

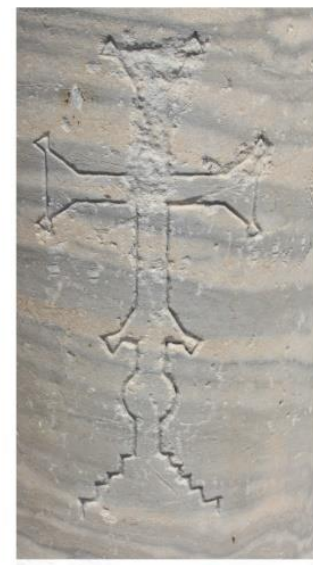

7

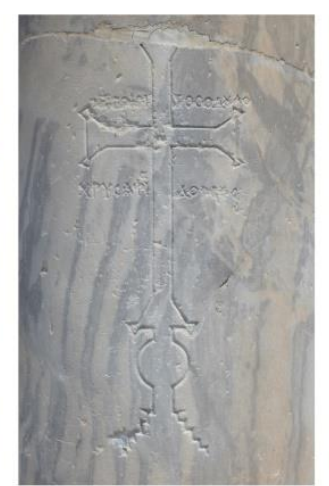

4

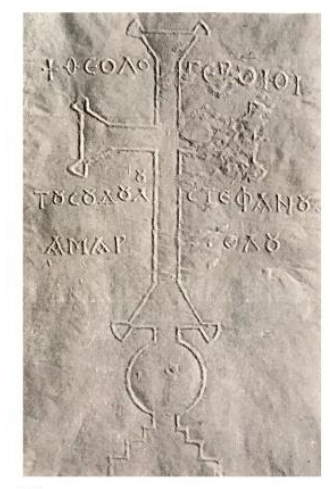

8

FIG. 14 - Foto d'insieme delle 8 colonne su cui sono incise le croci e le rispettive iscrizioni (foto S. Pedone).

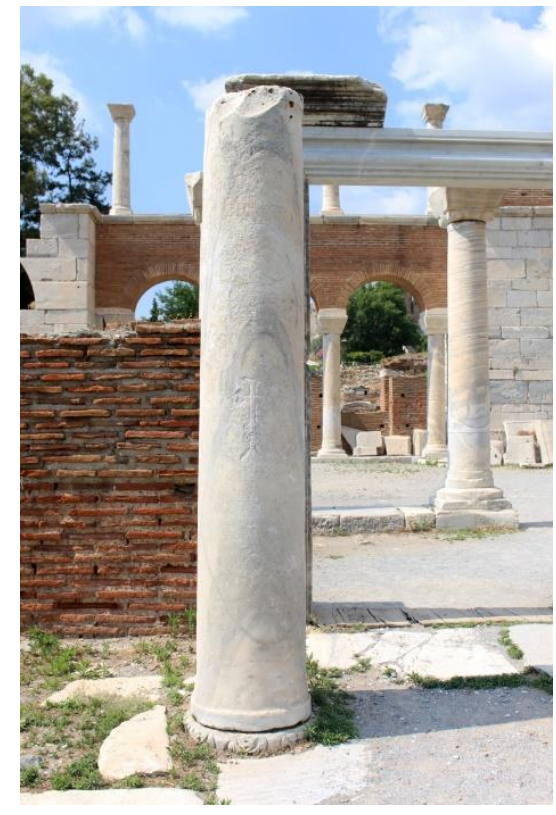

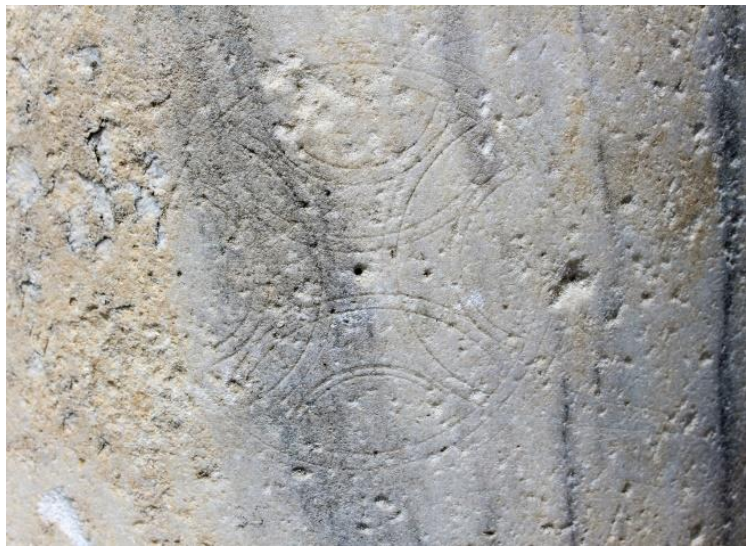

FIG. 15 - Efeso, Basilica di San Giovanni, colonna 1 (foto S. Pedone).

FIG. 16 - Efeso, Basilica di San Giovanni, colonna 2 (foto S. Pedone). 


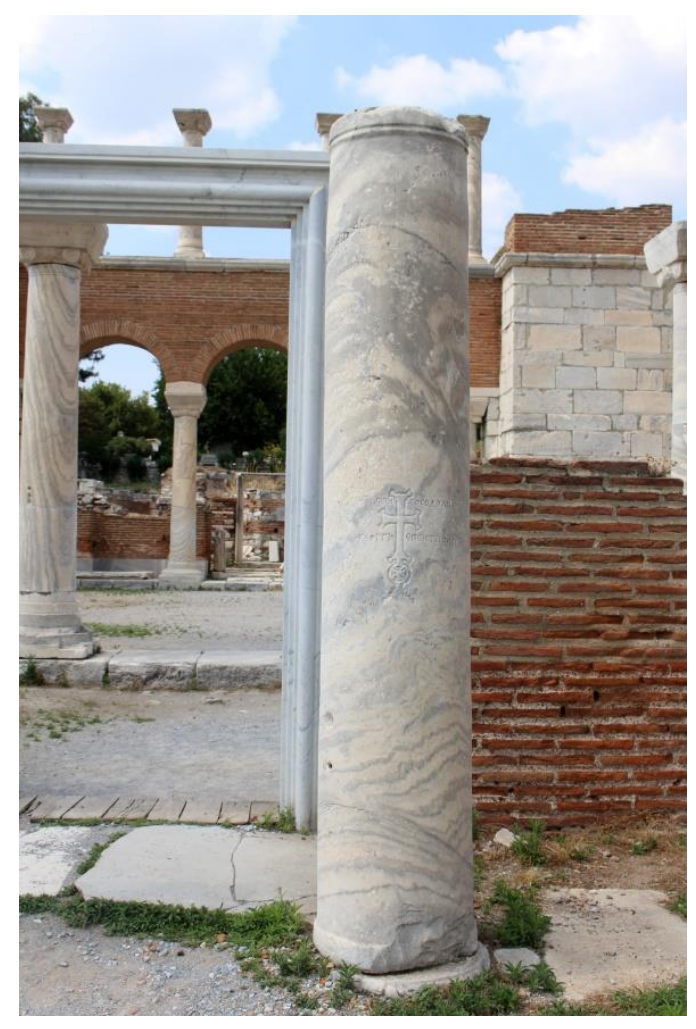

FIG. 17 - Efeso, Basilica di San Giovanni, colonna 3 (foto S. Pedone).

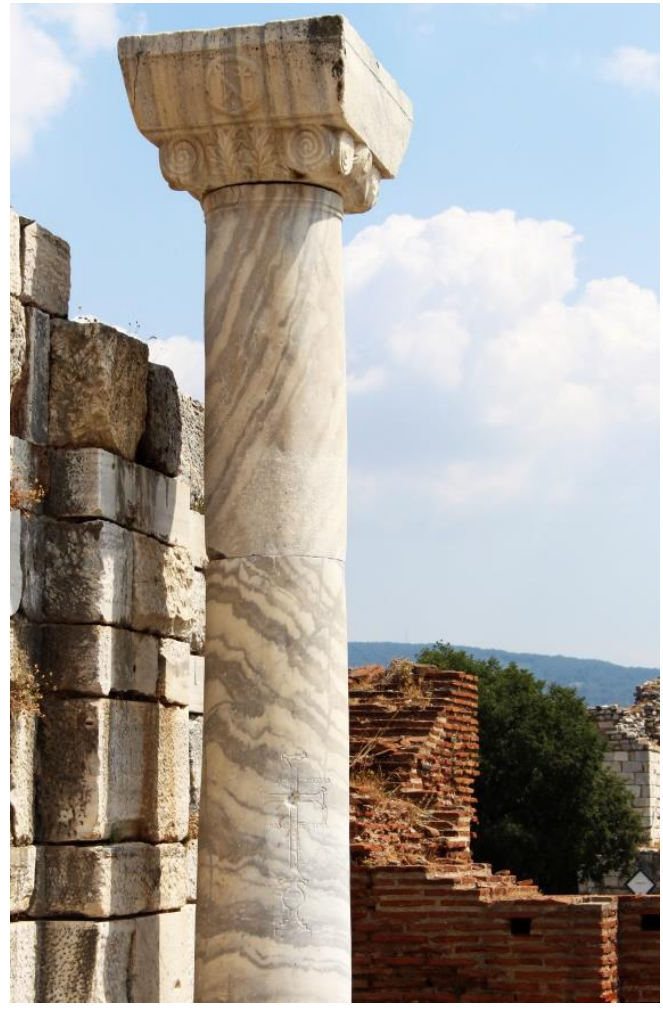

FIG. 18 - Efeso, Basilica di San Giovanni, colonna 4 (foto S. Pedone). 


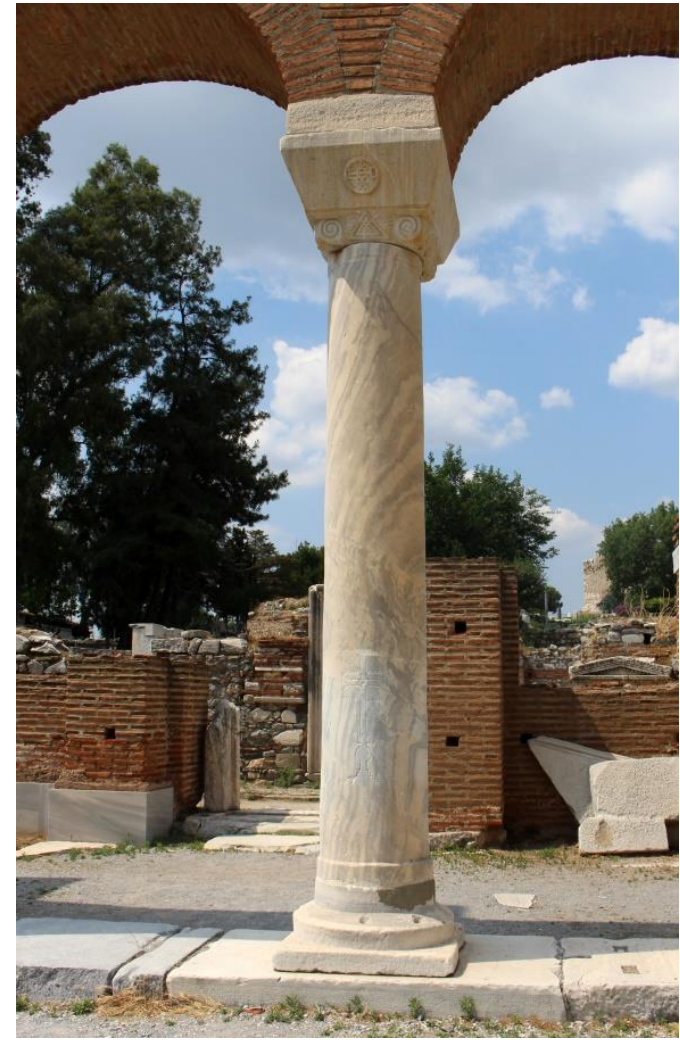

FIG. 19 - Efeso, Basilica di San Giovanni, colonna 5 (foto S. Pedone).

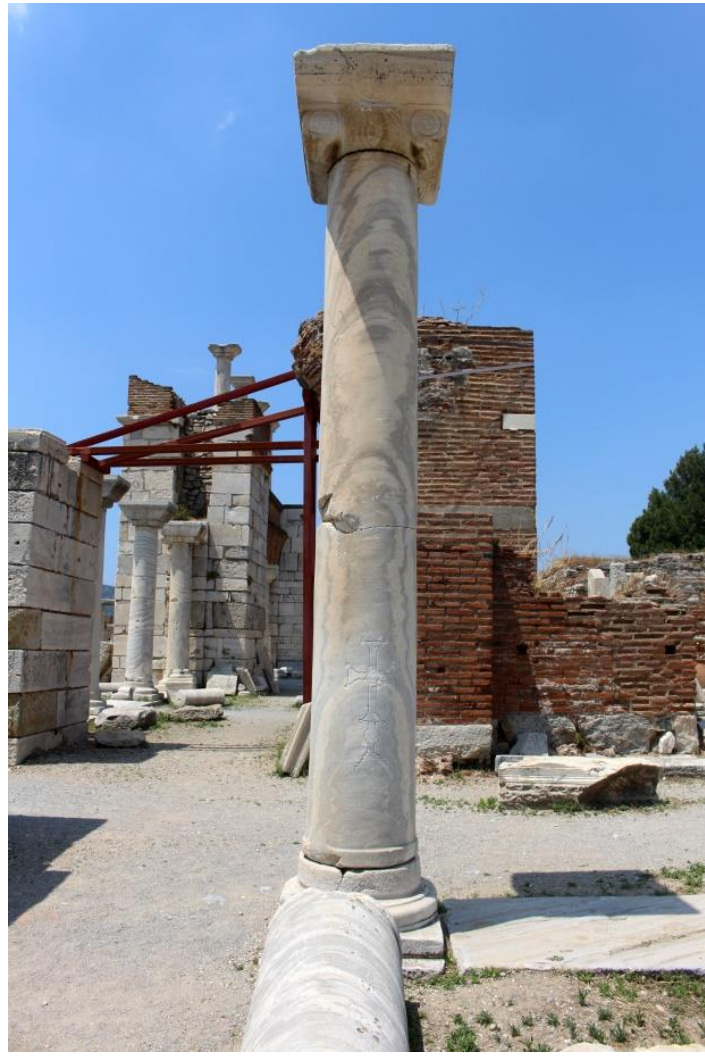

FIG. 20 - Efeso, Basilica di San Giovanni, colonna 6 (foto S. Pedone). 


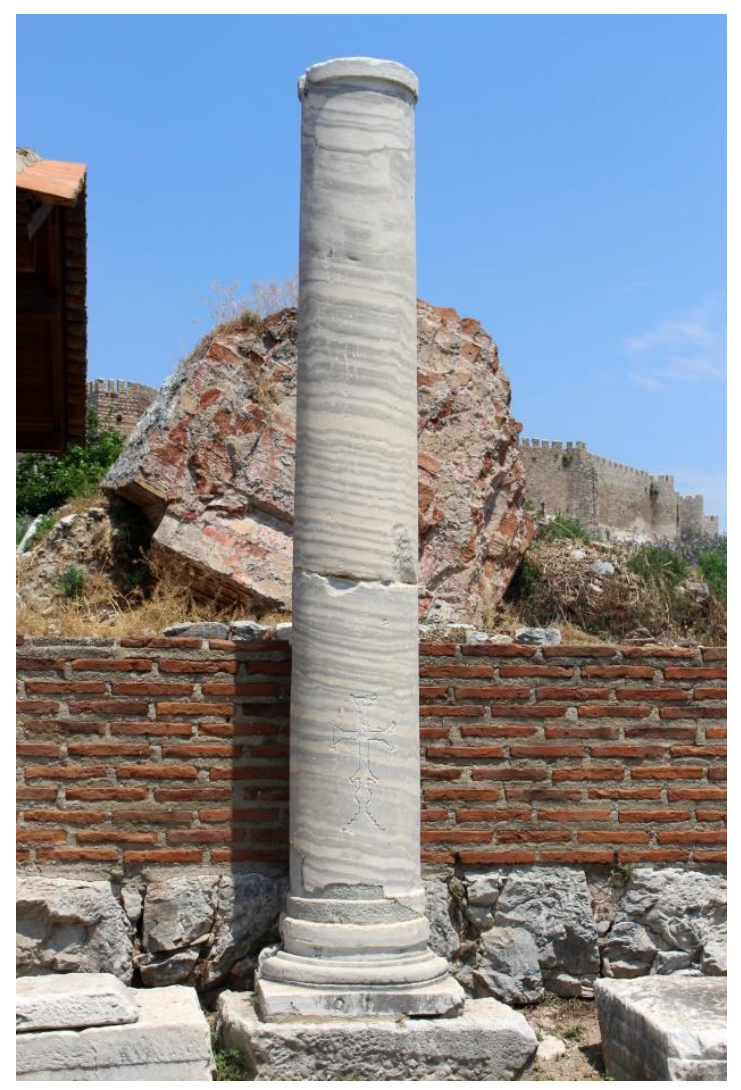

FIG. 21 - Efeso, Basilica di San Giovanni, colonna 7 (foto S. Pedone).

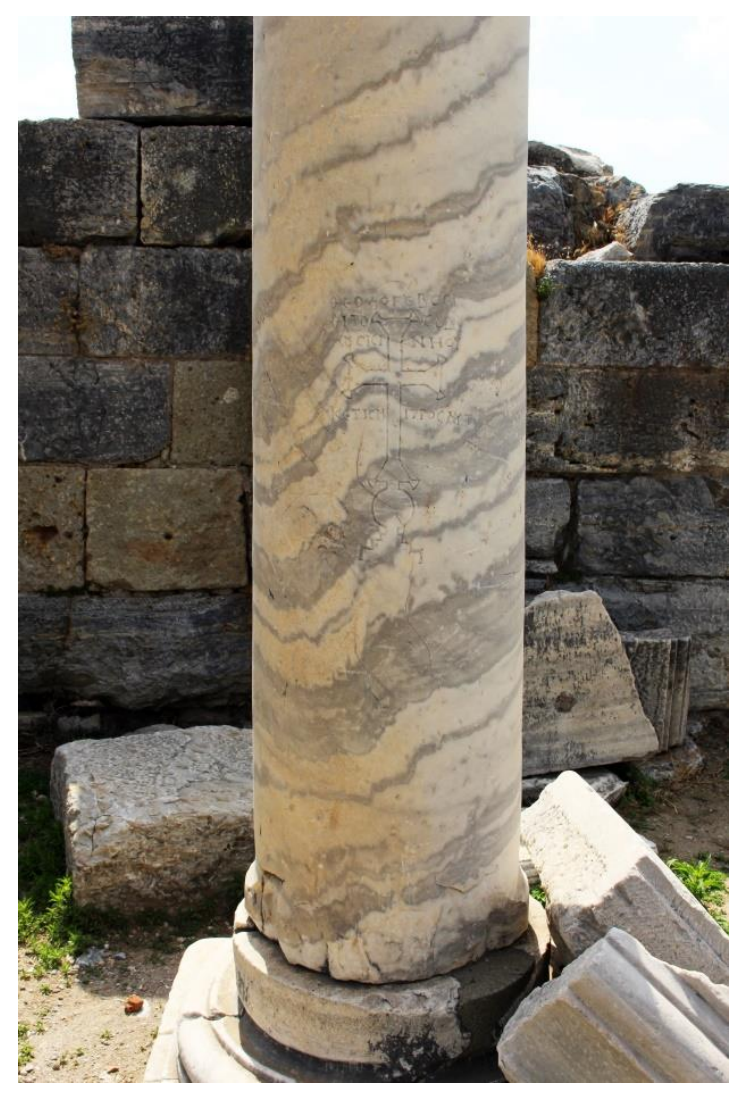

FIG. 22 - Efeso, Basilica di San Giovanni, colonna 8 (foto S. Pedone). 